\title{
Simulación del Modelo Matemático de la Cinemática Diferencial de Robots Seriales Planos Configuración RRR y RPR
}

\section{Simulation of the Mathematical Model of the Differential Kinematics of Serial Robots for the RRR and RPR Configuration}

\author{
MARTINEZ-ZAMUDIO, Patricio†*, GONZALEZ-VILLELA, Victor J. y LEON-NUÑEZ, Hector \\ Universidad Nacional Autónoma de México
}

ID $1^{\text {er }}$ Autor: Patricio, Martinez-Zamudio / ORC ID: 0000-0003-3378-5206, CVU CONACYT ID: 241062

ID $1^{\text {er }}$ Coautor: Victor J., Gonzalez-Villela / ORC ID: 0000-0002-6627-1204, CVU CONACYT ID: 262253

ID $2^{\text {do }}$ Coautor: Hector, Leon-Nuñez / ORC ID: 0000-0002-9745-6949, CVU CONACYT ID: 999106

DOI: $10.35429 /$ JSL.2019.20.6.1.8

Recibido 26 Junio, 2019; Aceptado 30 Septiembre, 2019

\begin{abstract}
Resumen
En este artículo se presenta el modelado y la simulación de dos configuraciones de robots seriales del tipo RRR y RPR, aplicando las teorías de la cinemática diferencial, para obtener la representación de su modelo matemático (matriz jacobiana) y su simulación. La cinemática diferencial en robótica es la relación entre dos espacios vectoriales, por lo cual es posible realizar el mapeo de velocidades del espacio articular al espacio de trabajo del efector final. Se presenta el modelo cinemático diferencial que se obtiene a partir de la cinemática de posición mediante técnicas de diferenciación y con ayuda de la matriz asimétrica se obtiene la información que forma parte de la matriz jacobiana, la cual permite conocer las velocidades de las variables articulares en función de la velocidad lineal y angular en el efector final y viceversa. Se realiza la simulación de los manipuladores validando el modelo matemático diferencial; A través de la validación de la cinemática diferencial de cadenas seriales simples es posible aplicar el procedimiento a robots manipuladores complicados. El método presentado aquí es la base de una herramienta útil para resolver robots complejos, como el caso de los robots manipuladores seriales redundantes, paralelos e híbridos.
\end{abstract}

Cinemática diferencial, Robótica, Matriz jacobiana

\begin{abstract}
This article presents the model and simulation of the serial robot configurations of the types RRR and RPR, applying the theories of differential kinematics, to obtain the representation of its mathematical model (Jacobian matrix) and its simulation. The differential kinematics in robotics is the relationship between vector spaces, so it is possible to make the velocity map in the joint space in the end effector workspace. We present the differential kinematic model that is obtained from the position kinematics by differentiation techniques and with the help of the asymmetric matrix we obtain the information that is part of the Jacobian matrix, which allows us to know the velocities of the joint variables as a function of linear and angular velocity in the end effector and vice versa. The simulation of the manipulators is carried out validating the mathematical differential model; through the validation of the differential kinematics of serial chains it is possible to apply the procedure to complicated manipulator robots. The method presented here is the basis of a useful tool for solving complex robots, as in the case of redundant, parallel and hybrid serial manipulator robots.
\end{abstract}

Differential kinematics, Robotics, Jacobian matrix

Citación: MARTINEZ-ZAMUDIO, Patricio, GONZALEZ-VILLELA, Victor J. y LEON-NUÑEZ, Hector. Simulación del Modelo Matemático de la Cinemática Diferencial de Robots Seriales Planos Configuración RRR y RPR. Revista de Simulación y Laboratorio 2019, 6-20: 1-8

*Correspondencia al Autor (Correo electrónico: pmz_rofi@ comunidad.unam.mx)

$\dagger$ Investigador contribuyendo como primer Autor 


\section{Introducción}

En algunas aplicaciones como el pintado por espray o soldadura es necesario realizar el análisis de velocidad, para mover al efector final por determinadas trayectorias con una velocidad prescrita, para lograr esta meta se deben de coordinar cuidadosamente las articulaciones del robot. Para que un robot serial siga una trayectoria, la velocidad debe ser continua en el extremo, esto sólo se logra a través de la coordinación de las variables articulares y el efector final, la velocidad que se describe en el efector final es constante, pero esto no ocurre en las variables articulares, por lo que es necesario obtener el modelado de la cinemática diferencial, obteniendo una matriz jacobiana, esta realiza la función de una transformación en espacios vectoriales de las variables articulares y el espacio vectorial de la velocidad en el efector final, (Tsai 1999).

La matriz jacobiana es una matriz formada por las derivadas parciales de primer orden. En robots manipuladores la matriz jacobiana o el jacobiano se define como una matriz que transforma las velocidades angulares al espacio de las velocidades del efector final. Diferentes métodos se han presentado en la literatura: en (Craig 2006) se presenta el método de propagación de velocidades; en (Featherstone 1983) se describe los procedimientos para realizar transformaciones de la posición y velocidad del efector final a los ángulos correspondientes, velocidades, y viceversa.

En (Hunt 1987) se examina el jacobiano de robots seriales por medio de la teoría de tonillos; en (Orin and Schrader 1984) se discuten y comparan seis métodos diferentes para calcular el Jacobiano de un robot manipulador de $N$ $G D L$; en (Waldron, Wang et al. 1985) se presenta una metodología general para la formulación y la manipulación de la matriz jacobiana; en (Whitney 1987) se da una nueva solución para el problema de control de posición del efector final.

En este trabajo se presenta el modelo cinemático diferencial que se obtiene a partir de la cinemática de posición mediante técnicas de diferenciación y con ayuda de la matriz asimétrica (Spong, Hutchinson et al. 2005), donde se obtiene la información que forma parte de la matriz jacobiana.
La cual permite conocer las velocidades de las variables articulares en función de la velocidad lineal y angular en el efector final y viceversa (Martínez-Zamudio, González-Villela et al. 2015).

\section{Cinemática diferencial}

En este trabajo la cinemática diferencial, se obtiene a partir del modelo matemático de la cinemática de posición (Tsai 1999) y aplicando el concepto de la matriz asimétrica (Barrientos, Cruz et al. 2007), se obtiene el modelado cinemático de los robots manipuladores seriales $R R R$ y $R P R$. Se realiza la implementación del modelo matemático de la cinemática diferencial validando el modelo a través de la simulación.

La cinemática diferencial de cadenas seriales $R R R$ y $R P R$ permiten conocer el comportamiento del manipulador. La simulación de las cadenas cinemáticas permite observar si el modelo es válido sin llevarlo a la implementación física. A través de la validación de la cinemática diferencial de cadenas seriales simples es posible aplicar el procedimiento a robots manipuladores complicados.

El método presentado aquí es la base de una herramienta útil para resolver la cinemática de robots complejos con ayuda de la solución de la cinemática directa e inversa, utilizando la integración de la cinemática diferencial, siendo un concepto novedoso e importante para los manipuladores de cadenas cinemáticas cerradas cuyas soluciones no existen, son difíciles de obtener, o son demasiado complejas para ser tratadas, por ejemplo: robots redundantes, paralelos e híbridos.

\section{Matriz jacobiana}

El Jacobiano establece la relación entre las velocidades articulares y la velocidad linear y angular en el extremo o efector final del robot (Merlet, et al. 2002), expresadas en el sistema inercial del robot, ver Ecuación (1).

$[\dot{X}]=J[\dot{q}]$

Para su obtención es necesaria la matriz de transformación homogénea que define el modelo cinemático directo del robot. 
La velocidad lineal del extremo del robot expresada desde el sistema inercial se obtiene mediante las derivadas respecto al tiempo de la sub matriz de posición obtenidas de la matriz de transformación homogénea resultado de la cinemática directa, (Barrientos, Cruz et al. 2007). Al obtener la sub-matriz de posición del efector final referido con el sistema inercial del manipulador serial, se obtiene una matriz de rotación dentro de la matriz de transformación homogénea, esta es una matriz ortonormal que contiene las rotaciones de los sistemas. Para obtener las velocidades angulares se deriva la submatriz de rotación (Barrientos, Cruz et al. 2007) para esto se hace uso de la matriz asimétrica, denominada como la matriz Omega $(\Omega)$, (Spong, Hutchinson et al. 2005) que proporciona las velocidades angulares del efector final referido al sistema inercial.

$\Omega=\dot{R} R^{T}$

La matriz asimétrica se define como (Spong, Hutchinson et al. 2005):

$\Omega=\left[\begin{array}{ccc}0 & -\Omega_{z} & \Omega_{y} \\ \Omega_{z} & 0 & -\Omega_{x} \\ -\Omega_{y} & \Omega_{x} & 0\end{array}\right]$

\section{Sistemas Multivariables}

De acuerdo con (Albertos and Antonio 2006), la definición de sistemas multi-variables es: "Si $p$ variables independientes son seleccionadas para ser controladas, por lo tanto, al menos el mismo número de variables independientes debe ser manipuladas $(m>=p)$ ". Se conoce como estado del sistema a la mínima cantidad de información necesaria en un instante para que, conociendo la entrada a partir de ese instante se pueda determinar cualquier variable del sistema en cualquier instante posterior.

$X(t)=S T(t) . U$

Donde $X$ representa a las variables de salida, $U$ las variables de entrada y $S T$ las ecuaciones a partir de las cuales es posible determinar las variables de estado. (Ogata 2003). Una forma de encontrar una representación de la cinemática del sistema $M I M O$ es mediante la realización de un mapeo de las velocidades de entrada $r_{m}$, donde $\eta(t)=[\eta 1, \ldots, \eta 2]$.
Estableciendo:

$$
\Sigma(q)=\left[\sigma_{m 1}(q), \ldots, \sigma_{m r_{m}}(q)\right]
$$

Es una matriz con los vectores de velocidades, parametrizada por las coordenadas angulares $\theta_{-}(i, j)$, expresamos el espacio de estados de las restricciones cinemáticas como:

$\dot{q}_{p}=\sum\left(\theta_{i, j}\right)[\eta]$

Donde $\eta$ es la matriz que representa la velocidad lineal y angular, del extremo del manipulador. Se define $A_{T}(q) \in R^{(M . N) x(3+M . N)}$ como la matriz asociada a la cinemática.

$$
A_{T}(q) \dot{q}=0
$$

Donde: $q \in R^{(3+M . N)}$ es el vector de las coordenadas de configuración y $\dot{q} \in R^{(3+M . N)}$ es el vector de las velocidades de configuración.

$\dot{q}_{p}=\left(\dot{x}_{p}, \dot{y}_{p}, \dot{\theta}_{p}, \dot{q}_{1,1}, \dot{q}_{1,2}, \ldots \dot{q}_{n, m}\right)$

Para solucionar el conjunto es conveniente seleccionar el conjunto de variables generalizadas que mejor convenga, para representar al sistema. Las velocidades del punto $P$ expresadas en coordenadas respecto al sistema inercial, así como la velocidad angular del efector final, en este caso describiendo los parámetros para un manipulador plano de $3 G D L$.

$\dot{q}_{p}=\left[S_{t n}\right][\eta]$

Donde $S_{t n}$ son vectores columna y $\eta=$ $\left[\begin{array}{lll}v_{x P} & v_{y P} & \omega_{P}\end{array}\right]$ es el vector de entradas. Por lo tanto:

$\dot{q}_{p}=\left[\begin{array}{lll}S_{t 1} & S_{t 2} & S_{t 3}\end{array}\right][\eta]$

De esta forma se tiene la cinemática del robot expresada en términos de las variables de entrada $\eta$.

$\left[\begin{array}{c}\dot{\mathrm{x}}_{\mathrm{p}} \\ \dot{\mathrm{y}}_{\mathrm{p}} \\ \dot{\theta p} \\ \dot{\mathrm{q}}_{1,1} \\ \vdots \\ \dot{\mathrm{q}}_{\mathrm{n}, \mathrm{m}}\end{array}\right]=\left[\begin{array}{ccc}1 & 0 & 0 \\ 0 & 1 & 0 \\ 0 & 0 & 1 \\ \mathrm{~S}_{\mathrm{t} 1,1} & \ldots & \mathrm{S}_{\mathrm{t} 1, \mathrm{~m}} \\ \vdots & \ddots & \vdots \\ \mathrm{S}_{\mathrm{tn}, 1} & \ldots & \mathrm{S}_{\mathrm{tn}, \mathrm{m}}\end{array}\right] \cdot\left[\begin{array}{ll}\eta & \end{array}\right]$ 


\section{Modelado e implementación de los casos particulares}

Se analizan dos robots seriales a partir de sus ecuaciones de estado; así como su implementación. El análisis se enfoca a los siguientes manipuladores, Robot serial plano (RRR) y Robot serial plano (RPR). Se decidió estudiar las configuraciones descritas para comprender el comportamiento y validar la teoría descrita, así como validar la hipótesis de este trabajo: demostrar que es posible controlar a un manipulador serial a partir de la cinemática diferencial.

\section{Manipulador serial RRR}

El manipular se encuentra compuesto por tres articulaciones rotacionales y tres eslabones. Cada articulación posee un actuador rotacional que genera el movimiento en el efector final $(x, y, \theta)$, donde $\theta$ es una rotación del efector final sobre el eje $z$. Se considera el espacio de trabajo sobre el plano, con $3 G D L$, de los cuales, dos grados de libertad logran posicionar al efector final a lo largo de los ejes $x, y$, y el tercero es una rotación alrededor del eje $z$. La Figura 1 muestra la postura del manipulador $R R R$. Donde $x, y$ es la posición del efector final respecto al sistema inercial y $\theta=\theta p$ es la orientación del efector final referido al sistema absoluto.

$$
\xi=(x, y, \theta)^{T}
$$

\section{Modelado cinemático}

Para encontrar las ecuaciones que describen la posición se utiliza la metodología de Denavit-Hartenberg (Cruz 2007). Aplicada a la Figura 1.

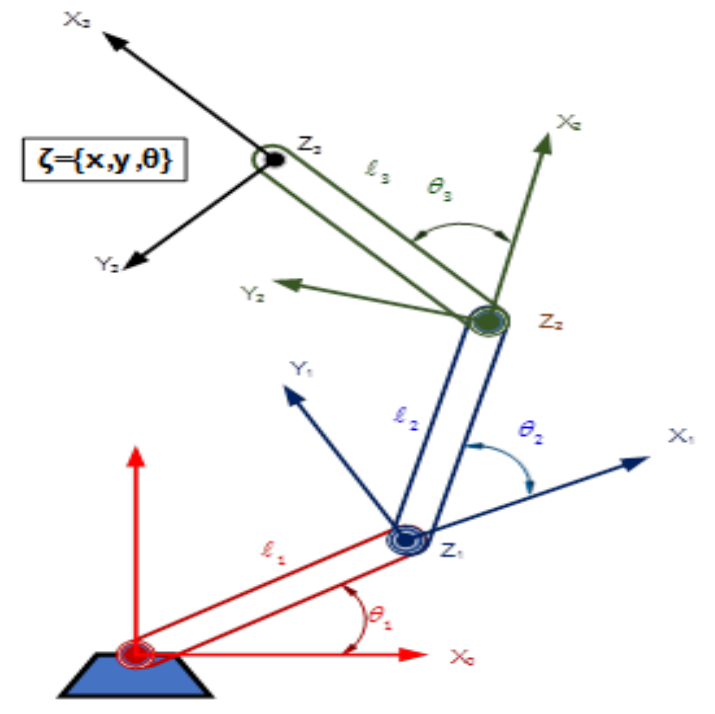

Figura 1 Postura del manipulador RRR

\begin{tabular}{|c|c|l|l|l|}
\hline $\boldsymbol{i}$ & $\boldsymbol{\theta}_{\mathbf{i}}$ & $\boldsymbol{d}$ & $\boldsymbol{a}_{\boldsymbol{i}}$ & $\boldsymbol{\alpha}_{\boldsymbol{i}}$ \\
\hline $\mathbf{1}$ & $\theta_{1}$ & 0 & $l_{1}$ & 0 \\
\hline $\mathbf{2}$ & $\theta_{2}$ & 0 & $l_{2}$ & 0 \\
\hline $\mathbf{3}$ & $\theta_{3}$ & 0 & $l_{3}$ & 0 \\
\hline
\end{tabular}

Tabla 1 Parámetros D-H manipulador RRR

La matriz de transformación que describe la posición del manipulador es la siguiente:

${ }^{0} A_{3}$
$=\left[\begin{array}{cccc}C_{123} & -S_{123} & 0 & C_{1} \ell_{1}+C_{12} \ell_{2}+C_{123} \ell_{3} \\ S_{123} & C_{123} & 0 & S_{1} \ell_{1}+S_{12} \ell_{2}+S_{123} \ell_{3} \\ 0 & 0 & 1 & 0 \\ 0 & 0 & 0 & 1\end{array}\right]$

\section{Cinemática diferencial}

Se utiliza el vector de posición que se extrae de la ecuación 15.

$X=C_{1} \ell_{1}+C_{12} \ell_{2}+C_{123} \ell_{3}$

$Y=S_{1} \ell_{1}+S_{12} \ell_{2}+S_{123} \ell_{3}$

Se obtiene el modelo cinemático diferencial, obteniendo las derivadas parciales de las ecuaciones (14), (15) con respecto a cada una de las variables articulares. La sub matriz de rotación se extrae de la ecuación (13), la cual se derivada, por ser una matriz ortogonal, guarda una relación con una matriz asimétrica(Spong, Hutchinson et al. 2005).

$$
{ }^{0} R_{3}=\left[\begin{array}{ccc}
C_{123} & -S_{123} & 0 \\
S_{123} & C_{123} & 0 \\
0 & 0 & 1
\end{array}\right]
$$

De la ecuación 2 se tiene:

$$
{ }^{0} \Omega_{3}={ }^{0} \dot{\mathrm{R}}_{3}{ }^{0} R_{3}^{T}
$$

Teniendo como matriz:

$$
\left[\begin{array}{ccc}
0 & -\left(\dot{\theta}_{1}+\dot{\theta}_{2}+\dot{\theta}_{3}\right) & 0 \\
\dot{\theta}_{1}+\dot{\theta}_{2}+\dot{\theta}_{3} & 0 & 0 \\
0 & 0 & 0
\end{array}\right]
$$

La matriz jacobiana del manipulador $R R R$ es la siguiente: 


$$
\left[\begin{array}{c}
\dot{\mathrm{V} x} \\
\mathrm{Vy} \\
\dot{\mathrm{V} z} \\
\omega \mathrm{x} \\
\omega \mathrm{y} \\
\omega \mathrm{z}
\end{array}\right]=\left[\begin{array}{ccc}
\frac{\partial x}{\partial \theta 1} & \frac{\partial x}{\partial \theta 2} & \frac{\partial x}{\partial \theta 3} \\
\frac{\partial y}{\partial \theta 1} & \frac{\partial y}{\partial \theta 2} & \frac{\partial y}{\partial \theta 3} \\
0 & 0 & 0 \\
0 & 0 & 0 \\
0 & 0 & 0 \\
1 & 1 & 1
\end{array}\right] \cdot\left[\begin{array}{c}
\dot{\theta}_{1} \\
\dot{\theta}_{2} \\
\dot{\theta}_{3}
\end{array}\right]
$$

\section{Implementación de la matriz del sistema multivariables del manipulador $R R R$}

El sistema del manipulador consta de múltiples entradas y múltiples salidas. La matriz del sistema en variables de estado se obtiene mediante la preselección de variables. El sistema consta de tres entradas en los dos casos particulares y con tres salidas, por tal motivo es necesario definir un vector de tres entradas para poder resolver el sistema. Las tres variables que se seleccionan para el vector de entradas son las velocidades de las variables articulares de los manipuladores, obteniendo el jacobiano de los manipuladores, dónde se relacionan estas velocidades articulares con las velocidades del extremo del robot.

Con las variables de las articulaciones se construyen tres ecuaciones que respectivamente cada una representa la velocidad en $X, Y$, la velocidad angular que se encuentra en $Z$, estas tres ecuaciones conforman un sistema de ecuaciones, el cual se resolvió para hallar a las variables articulare que fue escogido como vector de entradas. El sistema de ecuaciones es ordenado de manera matricial de tal forma que se obtiene una matriz de tres columnas por seis filas, la cual contiene la información para el mapeo de las velocidades. La implementación de este sistema se basó en las ecuaciones obtenidas por el análisis cinemático diferencial. La matriz del sistema en variables de estado del manipulador $R R R$ es:

$$
S_{t 1 R R R}=\left[\begin{array}{c}
1 \\
0 \\
0 \\
\frac{C_{12} \cdot C s c_{2}}{\ell_{1}} \\
C s c_{2}\left(-\frac{C_{12}}{\ell_{1}}-\frac{C_{1}}{\ell_{2}}\right) \\
\frac{C_{1} \cdot C s c_{2}}{\ell_{2}}
\end{array}\right]
$$

$$
\begin{aligned}
& S_{t 2 R R R}=\left[\begin{array}{c}
0 \\
1 \\
0 \\
C s c_{2} \cdot S_{12} \\
\ell_{1} \\
C s c_{2}\left(-\frac{s_{12}}{\ell_{1}}-\frac{s_{1}}{\ell_{2}}\right) \\
\frac{C s c_{2} \cdot S_{1}}{\ell_{2}}
\end{array}\right] \\
& 0 \\
& 0 \\
& 1 \\
& S_{t 3 R R R}=\left[\begin{array}{c}
C s c_{2} \cdot S_{3} \ell_{3} \\
\ell_{1} \\
-\frac{C s c_{2}\left(S_{12} \ell_{1}+S_{3} \ell_{2}\right) \ell_{3}}{\ell_{1} \ell_{2}} \\
1+\frac{C s c_{2} \cdot S_{12} \ell_{3}}{\ell_{2}}
\end{array}\right]
\end{aligned}
$$

Sustituyendo la Ecuación (20-22) en la Ecuación (9) se tiene:

$\dot{q}_{p R R R}=\left[\begin{array}{lll}S_{t 1 R R R} & S_{t 2 R R R} & S_{t 3 R R R}\end{array}\right]\left[\eta_{R R R}\right]$

Donde:

$$
\eta_{R R R}=\left[\begin{array}{lll}
V x & V y & \omega z
\end{array}\right]^{T}
$$

La Ecuación (23), es la ecuación que describe al modelo matemático de la cinemática diferencial del manipulador $R R R$. La cual se utiliza para realizar la simulación.

\section{Manipulador serial $R P R$}

El manipulador se encuentra compuesto por dos articulaciones rotacionales, una prismática y tres eslabones. Su área de trabajo actúa en el plano, con $3 G D L$, de los cuales, dos grados de libertad logran posicionar al efector final a lo largo de los ejes $x, y$, el tercero es una rotación alrededor del eje $z$. Ver Figura 2.

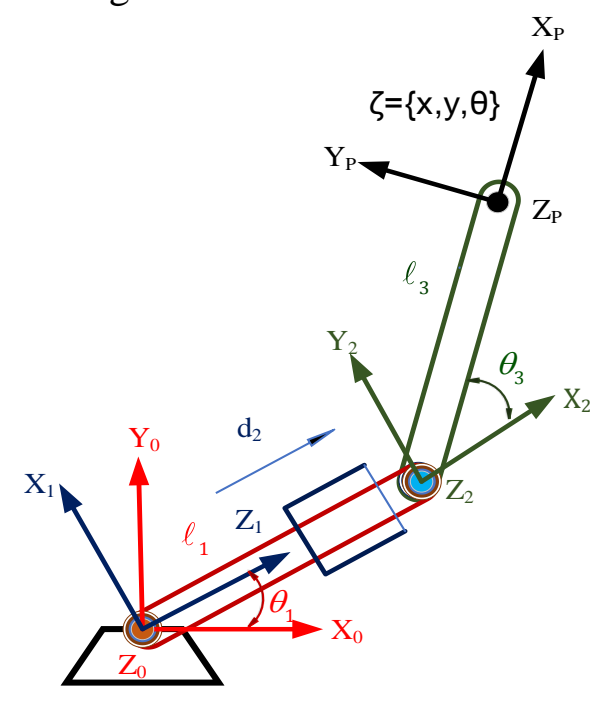

Figura 2 Postura del manipulador RPR

MARTINEZ-ZAMUDIO, Patricio, GONZALEZ-VILLELA, Victor J. y LEON-NUÑEZ, Hector. Simulación del Modelo Matemático de la Cinemática Diferencial de Robots Seriales Planos Configuración RRR y RPR. Revista de Simulación y Laboratorio 2019. 


\section{Modelado cinemático}

Para encontrar las ecuaciones que describen la posición se utiliza la metodología de DenavitHartenberg.

\begin{tabular}{|c|c|l|l|c|}
\hline $\boldsymbol{i}$ & $\boldsymbol{\theta}_{\mathbf{i}}$ & $\boldsymbol{d}$ & \multicolumn{1}{c|}{$\boldsymbol{a}_{\boldsymbol{i}}$} & $\boldsymbol{\alpha}_{\boldsymbol{i}}$ \\
\hline $\mathbf{1}$ & $\theta_{1}+90^{\circ}$ & 0 & 0 & $90^{\circ}$ \\
\hline $\mathbf{2}$ & 0 & $l_{1}+\mathrm{d}_{2}$ & 0 & $-90^{\circ}$ \\
\hline $\mathbf{3}$ & $\theta_{3}$ & 0 & $l_{3}$ & 0 \\
\hline
\end{tabular}

Tabla 2 Parámetros D-H manipulador $R P R$

La matriz de transformación que describe la posición del manipulador es la siguiente:

$$
\begin{aligned}
& \quad{ }^{0} A_{3} \\
& =\left[\begin{array}{cccc}
-S_{13} & -C_{13} & 0 & C_{1}\left(\ell_{1}+\mathrm{d}_{2}\right)-S_{13} \ell_{3} \\
C_{13} & -S_{13} & 0 & S_{1}\left(\ell_{1}+\mathrm{d}_{2}\right)+C_{13} \ell_{3} \\
0 & 0 & 1 & 0 \\
0 & 0 & 0 & 1
\end{array}\right]
\end{aligned}
$$

\section{Cinemática diferencial}

Se extrae el vector de posición de la Ecuación (25). Se obtiene el modelo cinemático diferencial, obteniendo las derivadas parciales de las ecuaciones del vector posición, con respecto a cada una de las variables articulares.

$$
\left[\begin{array}{c}
\dot{\mathrm{V}} \\
\mathrm{Vy} \\
\dot{\mathrm{V} z} \\
\omega \mathrm{x} \\
\omega \mathrm{y} \\
\omega \mathrm{z}
\end{array}\right]=\left[\begin{array}{ccc}
\frac{\partial x}{\partial \theta 1} & \frac{\partial x}{\partial d 2} & \frac{\partial x}{\partial \theta 3} \\
\frac{\partial y}{\partial \theta 1} & \frac{\partial y}{\partial d 2} & \frac{\partial y}{\partial \theta 3} \\
0 & 0 & 0 \\
0 & 0 & 0 \\
0 & 0 & 0 \\
1 & 0 & 1
\end{array}\right] \cdot\left[\begin{array}{c}
\dot{\theta}_{1} \\
\dot{d}_{2} \\
\dot{\theta}_{3}
\end{array}\right]
$$

La sub matriz de rotación se extrae de la ecuación (25), la cual se deriva. Por ser una matriz ortogonal, guarda una relación con una matriz asimétrica. (Spong, Hutchinson et al. 2005).

\section{Implementación de la matriz del sistema multivariables del manipulador $R P R$}

El sistema del manipulador consta de múltiples entradas y múltiples salidas. La matriz del sistema en variables de estado se obtiene mediante la selección de variables. El sistema consta de tres entradas en los dos casos particulares y con seis salidas, por tal motivo es necesario definir un vector de entradas para poder resolver el sistema.
Las tres variables que se seleccionan para el vector de entradas son las velocidades en las variables articulares de los manipuladores, con el jacobiano obtenido de los manipuladores, dónde se encuentra la relación de velocidades del extremo del robot. Con las variables de las articulaciones se construyen tres ecuaciones que respectivamente cada una representa la velocidad en $X, Y$, la velocidad angular que se encuentra en $Z$, estas tres ecuaciones conforman un sistema de ecuaciones el cual se resolvió para hallar a las variables articulare. El sistema de ecuaciones es ordenado de manera matricial de tal forma que se obtiene una matriz de tres columnas por seis filas la cual contiene la información para el mapeo de las velocidades. La implementación de este sistema se basó en las ecuaciones obtenidas por el análisis cinemático diferencial. La matriz del sistema en variables de estado del manipulador $R P R$.

$$
\dot{q}_{p R P R}=\left[\begin{array}{lll}
S_{t 1 R P R} & S_{t 2 R P R} & S_{t 3 R P R}
\end{array}\right]\left[\eta_{R P R}\right]
$$

Donde:

$$
\eta_{R P R}=\left[\begin{array}{lll}
V x & V y & \omega z
\end{array}\right]^{T}
$$

La Ecuación (27), es la Ecuación que describe al modelo matemático de la cinemática diferencial del manipulador $R P R$. La cual se utiliza para realizar la simulación.

\section{Simulación del manipulador $R R R$ y $R P R$}

El modelo matemático de la cinemática diferencial describe la velocidad lineal del efector final en función de las variables articulares, así como la velocidad angular de este Ecuación (11). La Ecuación 11 es una forma general de la matriz de variables de estado. Esta matriz puede variar, es decir puede ser diferente, aunque el manipulador sea el mismo. 


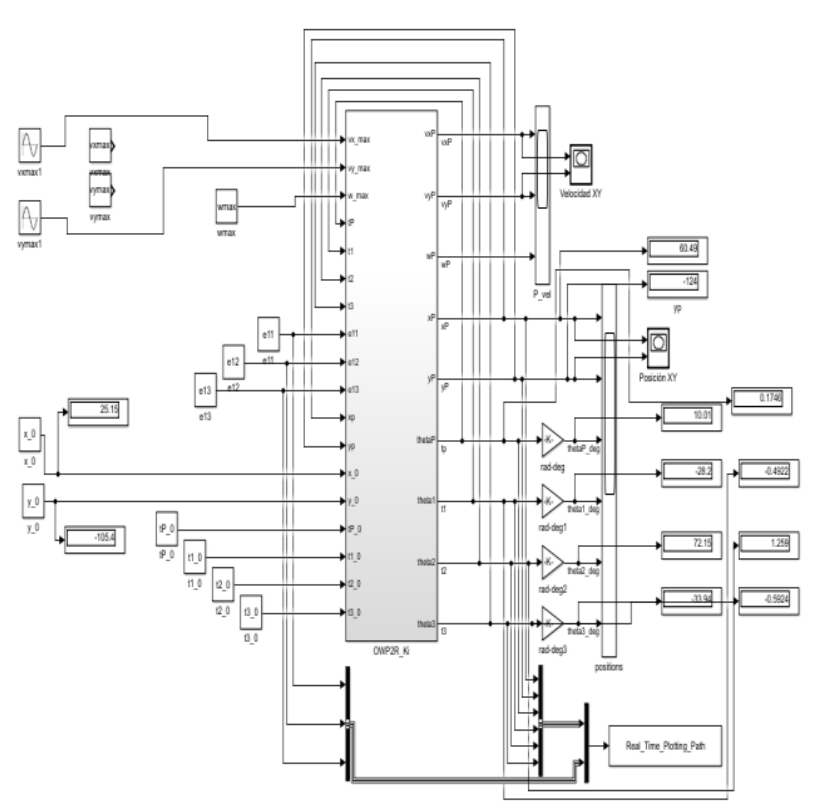

Figura 3 Diagrama de bloques entradas y salidas RRR y RPR

La simulación se llevó a cabo en Simulink con un diagrama de bloques que representa a cada elemento del manipulador y que despliega de forma gráfica el comportamiento del manipulador y la trayectoria generada por las velocidades de entrada, el diagrama de bloques se muestra a en la Figura 3. La trayectoria seleccionada para este manipulador es una lemniscata (ver Figura 4) creada con funciones senoidales, esto quiere decir que se selecciona un módulo de velocidad lineal en las dos componentes que responda a una función senoidal y una velocidad angular nula.

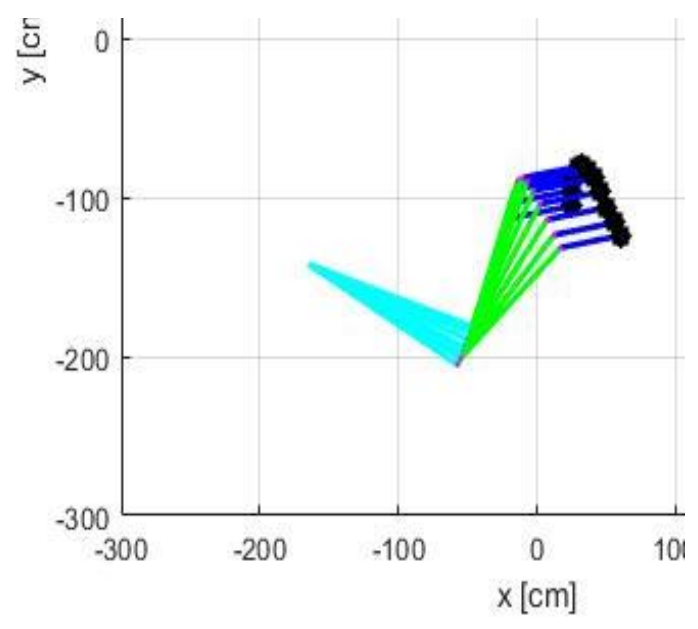

Figura 4 Trayectoria del manipulador RRR lemniscata

En la simulación se muestra el manipulador y su trayectoria, los eslabones son presentados en diferentes colores para no confundirse, la trayectoria esta simbolizada por puntos negros donde el efector final coincide y validamos el modelo cinemático diferencial.

\section{Simulación del manipulador RPR}

El efector final del manipulador coincide con los puntos de la trayectoria, esto quiere decir que el modelo diferencial es válido, así como la matriz del sistema, ver Figura 5.

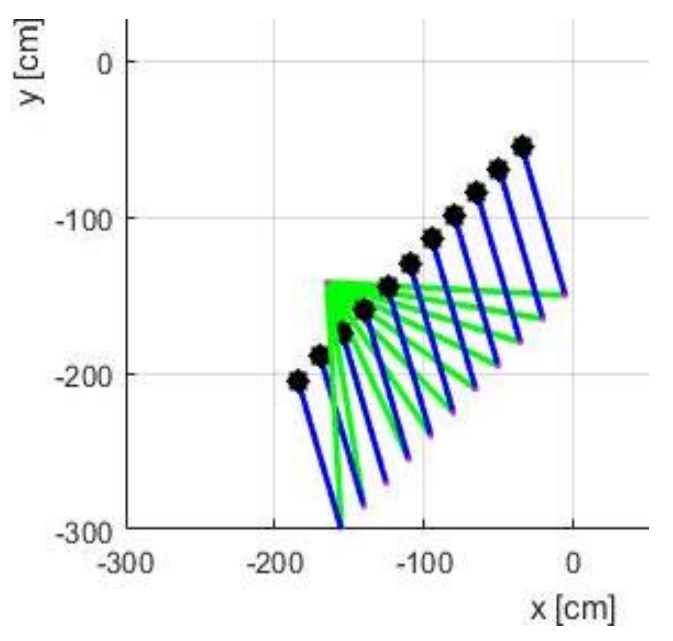

Figura 5 Trayectoria del manipulador RPR línea recta

En la segunda prueba los parámetros de entrada son reemplazados para obtener una velocidad constante en la parte lineal pero la velocidad angular es establecida como nula. El manipulador tiene que describir una línea recta como trayectoria, los parámetros de los eslabones son los mismos, ya que esto no afecta en los resultados. Los resultados obtenidos son satisfactorios, el modelo diferencial converge, el jacobiano, proporciona una matriz de velocidades angulares la cual debe ser asimétrica, esto permite visualizar si hay algún error en la matriz.

\section{Conclusiones}

Se presentó el modelo cinemático diferencial de manipuladores seriales con un enfoque diferente al que se presenta en la literatura, ya que en este trabajo se obtuvo el modelo a partir de la cinemática de posición, mediante técnicas de diferenciación y con ayuda de la matriz asimétrica. Se validó el modelo matemático de la cinemática diferencial por medio de la simulación de dos manipuladores seriales del tipo RRR y RPR. La simulación se llevó a cabo utilizando diferentes parámetros de entrada en el manipulador, generando así diferentes patrones de movimiento o trayectorias a seguir. Se utilizaron velocidades senoidales, constantes y variaciones dentro de estas. 
Las pruebas proporcionaron que el comportamiento del extremo del robot coincidía con la trayectoria generada, esto valida los modelos diferenciales obtenidos de las Ecuaciones (23 y 27). La validación de la cinemática diferencial de cadenas seriales simples mediante el método mostrado en este trabajo, augura la posibilidad de aplicar este el procedimiento a robots manipuladores complejos, como el caso de los robots manipuladores seriales redundantes, paralelos e híbridos.

\section{Agradecimiento}

Agradezco en lo que corresponde a la DGAPA, por el apoyo brindado para la realización de este trabajo, a través del proyecto UNAM-DGAPAPAPIME-PE112319: "Desarrollo de material didáctico y banco de pruebas para el fortalecimiento de la enseñanza en instrumentación y control, aplicando tecnologías IoT y Robótica."

\section{Referencias}

Albertos, P. and S. Antonio (2006). Multivariable Control Systems: An Engineering Approach, Springer London.

Barrientos, A., et al. (2007). Fundamentos de robótica, McGraw-Hill.

Craig, J. J. (2006). Robótica, Pearson Educación de México.

Cruz, A. B. (2007). Fundamentos de robótica, McGraw-Hill.

Featherstone, R. (1983). "Position and velocity transformations between robot end-effector coordinates and joint angles." The International Journal of Robotics Research 2(2): 35-45.

Hunt, K. H. (1987). "ROBOT KINEMATICS A COMPACT ANALYTIC INVERSE SOLUTION FOR VELOCITIES." Journal of mechanisms, transmissions, and automation in design 109(1): 42-49.

Martínez-Zamudio, P., et al. (2015). "Cinemática Diferencial de un Manipulador Paralelo Plano 3RRR-(RRR)v con Actuación Virtual Indirecta." Ingeniería mecánica, tecnología y desarrollo 5: 321-331.
Merlet, J. P. (2006). Parallel Robots, Springer. Merlet, J. P., et al. (2002). Computational Kinematics: Proceedings, INRIA.

Ogata, K. (2003). Ingeniería de control moderna, Pearson Educación.

Orin, D. E. and W. W. Schrader (1984). "EFFICIENT COMPUTATION OF THE JACOBIAN FOR ROBOT MANIPULATORS." International Journal of Robotics Research 3(4): 66-75.

Spong, M. W., et al. (2005). Robot Modeling and Control, Wiley.

Spong, M. W. and M. Vidyasagar (1989). Robot Dynamics and Control, Wiley.

Tsai, L. W. (1999). Robot Analysis: The Mechanics of Serial and Parallel Manipulators, Wiley.

Waldron, K. J., et al. (1985). "STUDY OF THE JACOBIAN MATRIX OF SERIAL MANIPULATORS." Journal of mechanisms, transmissions, and automation in design 107(2): 230-238.

Whitney, D. E. (1987). "HISTORICAL PERSPECTIVE AND STATE OF THE ART IN ROBOT FORCE CONTROL." International Journal of Robotics Research 6(1): 3-14. 